\title{
Evaluación económica como complemento necesario para una mejor valoración del manejo del riesgo
}

\author{
Economic assessment as necessary supplement for a better \\ assessment of risk management
}

\author{
Delia Mercedes Herrera Travieso, ${ }^{\mathrm{I}}$ Anaí García Fariñas ${ }^{\mathrm{II}}$ \\ IDoctor en Medicina. Máster en Salud Ambiental. Especialista de I Grado en Higiene \\ y Epidemiología. Instituto Nacional de Higiene, Epidemiología y Microbiología. La \\ Habana, Cuba. \\ II Licenciada en Ciencias Farmacéuticas. Doctor en Ciencias de la Salud. Máster en \\ Economía de la Salud. Escuela Nacional de Salud Pública. La Habana, Cuba.
}

\section{RESUMEN}

Se profundiza en la necesidad de articular las evaluaciones económicas con los estudios de evaluación de riesgo, en aras de lograr una mejor valoración de las medidas propuestas por estas últimas. Para ello se utilizó el ejemplo de la fuente fija de contaminación atmosférica de la Empresa Suchel Debón. Los cambios instrumentados en esta empresa para el control de la fuente representaron un costo directo total que superó el medio millón de pesos cubanos. Ante esta situación hubiese sido pertinente contar con una evaluación económica que permitiera valorar la relación entre esos costos y los resultados en salud, vistos estos últimos tanto desde la perspectiva del sector de la salud como del medio ambiente.

Palabras clave: Evaluación económica, manejo de riesgo, contaminación atmosférica, fuentes fijas de contaminación atmosférica.

\section{ABSTRACT \\ The need of integrating the economic evaluations and the risk assessment studies with a view to achieving a better evaluation of the measures suggested by the}


latter was deeply discussed. To this end, the example of the fixed source of environmental pollution of Suchel Debón Company was used. The implemented changes in this company for the control of the polluting source represented a total direct cost of more than 500000 Cuban pesos. In this type of situation, it would have been advisable to have an economic evaluation in place, which would have allowed assessing the relationship between these costs and the results in the field of health, considered from both the health sector perspective and the environmental perspective.

Key words: Economic evaluation, risk management, environmental pollution, fixed sources of environmental pollution.

\section{INTRODUCCIÓN}

Los efectos de la contaminación suelen ser muy costosos, más para una sociedad en desarrollo, en términos de residuos, degradación del medio ambiente, costo de limpieza en el futuro, así como la pérdida de la salud y la reducción de la calidad de vida. ${ }^{1}$

Cuando los riesgos para la salud de un peligro ambiental específico o de una situación han sido caracterizados, para tomar decisiones hay que tener en cuenta cuál de las diversas acciones de control deberán adoptarse. El conjunto de acciones y decisiones que se acometen para el control del riesgo conforman la base del proceso de manejo de riesgos. ${ }^{2,3}$

Frecuentemente los recursos necesarios para acometer estas acciones resultan limitados. Para ello es necesario evaluar la relación recursos empleados-resultados obtenidos, para cada una de las acciones o estrategias posibles a desarrollar como parte del manejo de los riesgos. ${ }^{4}$ Los estudios de evaluación económica han demostrado ser útiles en propiciar una decisión tomada en base a los beneficios potenciales en salud ambiental en relación con los costos que pueden ser asumidos. En Cuba, las evaluaciones económicas en salud son escasas y su relación con la toma de decisiones todavía es limitada. ${ }^{4-6}$

\section{EL CASO DE LA EMPRESA SUCHEL DEBÓN}

La planta de detergente de la empresa Suchel Debón fue identificada a principios de los años 2000 como una de las fuentes fijas de contaminación atmosférica más importante de La Habana, en especial para el municipio Cerro. La planta emitía a la atmósfera cantidades muy superiores a las permitidas de polvo de detergente, lo cual no solo afectaba la productividad de la entidad sino que ocasionaba malestar en la población. Como consecuencia, durante el año 2001 se realizaron cambios en la tecnología de la planta para disminuir las emisiones. Entre ellas se destacan, la sustitución del ácido sulfónico ramificado no biodegradable por el ácido sulfónico lineal biodegradable, la sustitución del separador húmedo de polvo de los gases de escape de la torre de secado por un filtro de mangas, la sustitución del horno de petróleo de la torre de secado por un horno de gas, la sustitución de los filtros de 
fundas del transportador neumático de polvo por filtros de mangas autolimpiables, la instalación de un sistema de transporte neumático de las materias primas en forma de polvo utilizadas en el proceso y la sustitución de los filtros de fundas del extractor de aire ambiental por filtros de mangas autolimpiables (Rodríguez López O. Trabajos realizados en la planta de detergente de la Empresa Suchel Debón para reducir la contaminación ambiental. Informe. Empresa Suchel Debón. Enero del 2006). Además se llevaron a cabo múltiples medidas de ingeniería, las que ayudaron a minimizar la exposición al peligro.

La planta de detergente desde sus inicios en el año 1950 utilizó el dodecilbenceno ramificado o tetrapropilbenceno no biodegradable, a finales del 2001 se sustituyó por el acido sulfónico lineal biodegradable para producir la materia activa del detergente en polvo, lo cual motivó una modificación en el proceso de neutralización de ese ácido, sustituyéndose los equipos instalados desde hace más de 50 años por un tanque reactor y una bomba dosificadora de tres cabezales. Esta sustitución no sólo eliminó la contaminación ambiental también mejoró la calidad del producto al obtenerse un polvo detergente más blanco y de mejor apariencia. Otra de las medidas fue en el sistema de depuración de los gases de escape de secado, se decidió sustituir el separador húmedo y el ciclón por un filtro de mangas, cuya norma operativa permitía hasta $50 \mathrm{mg} / \mathrm{m}^{3}$ de aire a la salida del filtro, con lo que se redujeron las emisiones de partículas de polvo de $18,2 \mathrm{mg} / \mathrm{m}^{3}$ en el año 2000 a $4,82 \mathrm{mg} / \mathrm{m}^{3}$ en el 2005.

La torre de secado de detergente utiliza aire caliente con una temperatura entre 300 y $400{ }^{\circ} \mathrm{C}$ para evaporar el agua de la pasta atomizada y convertirla en polvo detergente. El aire era calentado por un horno que empleaba petróleo como combustible, el cual se sustituyó por un nuevo horno que utiliza como combustible el gas licuado, con ello la emisión de partículas de hollín a la atmósfera se eliminó totalmente. Con los cambios realizados en la planta se logró una notable reducción de la emisión total de partículas de polvo a la atmósfera. De $197,7 \mathrm{mg} / \mathrm{m}^{3}$ partículas de polvo que se emitían a la atmósfera antes del año 2000 se redujo hasta 18,2 en el 2000.

Tecnológicamente las medidas tomadas se corresponden con lo establecido. Cinco de las seis acciones acometidas para el manejo del riesgo en la planta se ubican en el primer grupo de acciones a valorar también conocidas como de "control en la fuente". ${ }^{2}$ Sin embargo, la relación existente entre el costo asumido para el manejo del riesgo en esta empresa y los resultados que se esperaban obtener tanto para el medio ambiente como para la salud de la comunidad, no fue posible establecerla. Si bien se pudo determinar que, por concepto de equipamiento sustituido o introducido, el costo directo de esta estrategia de manejo de riesgo ascendió a 766 000,00 pesos cubanos; lo referido a la contratación de mano obra no fue posible contabilizarla por no encontrase la información primaria.

La mayoría de los registros de la empresa sobre los costos de inversión, sobre todo los relacionados con los recursos humanos y algunos recursos materiales no estaban preservados. A ello se unió la pobre información existente relativa a la demanda de servicios de salud debido a causas de morbilidad potencialmente relacionada con las emisiones de la planta ni de los costos que estas representan para el Sistema Nacional de Salud. Desafortunadamente no existe un estudio nacional previo de la correlación entre valores crecientes de partículas grandes que miden entre 2,5 y 10 micrómetros $\left(\mathrm{PM}_{10}\right)$ y el aumento de la demanda de servicios de salud, por ejemplo, los casos de infecciones respiratorias agudas y crisis agudas de asma bronquial, ${ }^{7-9}$ debido a ello también quedaron truncas las posibilidades de acercase a la relación recursos-resultados a partir de la construcción de un modelo teórico. 


\section{La relación costo-resultado como acercamiento a la eficiencia de las soluciones}

En la actualidad, es reconocido por cada vez un mayor número de países, la necesidad de complementar el proceso de toma de decisiones en salud con información relativa a la eficiencia. Al hablar de eficiencia, obligatoriamente ha de contarse con información sobre los recursos (comúnmente medidos a través de los costos) y sobre los resultados. Respecto a los costos es necesario insistir en que no solo debe considerarse el costo derivado del consumo de los recursos implicados en las medidas de control, sino que también deben medirse los costos evitados, es decir, el ahorro de recursos como consecuencia de una determinada decisión.

En el contexto de manejo de riesgo, una adecuada evaluación de la relación recursos-resultados implica tomar en consideración no solo el costo directo de las medidas implementadas y los efectos positivos logrados sobre el medio ambiente, sino que se necesita valorar los costos evitados por atenciones médicas en unidades del nivel primario y secundario, así como los evitados por disminución de las pérdidas de productividad.

El sector de la salud tiene mucho que aportar en la valoración de la eficiencia de las medidas que se toman para el manejo del riesgo de las diferentes fuentes de contaminación, pues buena parte de los efectos negativos de estas se expresan en cambios en la situación de salud de las comunidades a consecuencia de lo cual se modifica el uso de los servicios de salud y con ello casi siempre se incrementan los gastos de las entidades prestadoras de estos servicios. El sector de la salud debe jugar un papel más activo en la generación de evidencias de los efectos que las medidas de control tienen sobre sus indicadores y actividades.

Se puede concluir que aprovechar las potencialidades de las evaluaciones económicas como complemento de la evaluación del manejo del riesgo todavía hoy es un reto para los profesionales cubanos. Todos los esfuerzos que a este respecto se realicen redundarán en el fortalecimiento de la conciencia económica del sector y en la batalla por la eficiencia a la cual nos ha convocado la dirección del país.

\section{REFERENCIAS BIBLIOGRÁFICAS}

1. Guidotti TL. El riesgo ambiental para la salud: Los países en desarrollo y la contaminación. En: Stellman JM, editor. Encyclopaedia of Occupational Health and Safety. 4th ed. Geneva: International Labour Organization; 1998. p. 11.

2. Yassi $A$, Kjellstrom $T$, de Kok $T$, Guidottií $T$. Evaluación de riesgo. Salud Ambiental Básica. Molina Esquivel E, editor versión en español. México. D.F.: PNUMA; 2002.

3. Romero Placeres M, Más Bermejo P, Lacasaña Navarro M, Téllez Rojo-Solis MM, Aguilar Valdés J, Romieu I. Contaminación atmosférica, asma bronquial e infecciones respiratorias agudas en menores de edad en Ciudad Habana. Salud Pública Mex. 2004;46(3):222-32.

4. Gutiérrez Zúñiga $C$, Hernández Peña $P$, Ortiz Guerrero $C$, Zurutuza Fernández R, Santos-Burgoa C. Evaluación económica y toma de decisiones en salud ambiental. Rev Saúde Pública.1994;28(2):154-5.

http://scielo.sld.cu 
5. Gálvez González AM. La evaluación económica en salud en Cuba. Instrumento para la toma de decisiones [tesis]. La Habana: Escuela Nacional de Salud Pública; 2004.

6. Ministerio de Salud Pública. Anuario estadístico. La Habana: MINSAP; 2001.

7. Molina Esquivel E, Brown Colás LA, Prieto Díaz V, Bonet Gorbea M, Cuellar Luna

L. Crisis de asma y enfermedades respiratorias agudas, contaminantes atmosféricos y variables meteorológicas en Centro Habana. Rev Cubana Med Gen Integr. $2001 ; 17(1): 10-20$.

8. Molina Esquivel E, Brown Colas LA, Prieto Díaz V. Contaminación atmosférica y prevalencia de asma en Centro Habana. Rev Cubana Hig Epidemiol [serie en Internet]. 2001 [citado 6 Mar 2008];39(1). Disponible en:

http://scielo.sld.cu/scielo.php?script=sci arttext\&pid=S156130032001000100001\&lng=es

9. Carbonell García I, Lambert Matos R. Interrelación de la epidemiología con la economía de salud. Rev Cubana Hig Epidemiol [serie en Internet]. 1996 [citado 28 Mar 2008];34(2). Disponible en:

http://bvs.sld.cu/revistas/hie/vol34 2 96/hie05296.htm

Recibido: 12 de marzo de 2009.

Aprobado: 14 de mayo de 2010.

Delia Mercedes Herrera Travieso. Instituto Nacional de Higiene, Epidemiología y Microbiología. Infanta No. 1158 e/ Clavel y Llinás. Centro Habana 10300. Ciudad de La Habana, La Habana, Cuba. Correo electrónico: deliaherrera@infomed.sld.cu 